\title{
A AUTO-ESTIMA EM ADOLESCENTES COM E SEM FISSURAS DE LÁBIO E/OU DE PALATO
}

\author{
Denise de Andrade 2 \\ Emília Luígia Saporiti Angerami $i^{3}$
}

Andrade D, Angerami ELS. A auto-estima em adolescentes com e sem fissuras de lábio e/ou de palato. Rev Latino-am Enfermagem 2001 novembro-dezembro; 9(6):37-41.

O auto-conceito é um determinante importante da auto-estima, entre homens e mulheres, isto nos fez refletir sobre o processo de reabilitação das pessoas portadoras de malformação. Utilizamos para mensurar o nível de auto-estima a escala de Janis e Field. Entrevistouse 608 adolescentes sendo 235 com e 373 sem fissuras de lábio e/ou de palato, entre idades de 17 a 20 anos e de ambos o sexos. Analisando os níveis de auto-estima concluímos que o grupo de adolescentes com fissuras se comporta de forma diferente do grupo sem fissura, ou seja, os portadores de fissuras exibem menores escores de auto-estima.

DESCRITORES: adolescência, auto-estima, fissura labial, fissura palatina

\section{THE SELF-ESTEEM OF ADOLESCENTS WITH AND WITHOUT A CLEFT LIP AND/OR PALATE}

Self-image is an important determinant of self-esteem among men and women, which makes us reflect about the process of rehabilitation of people with facial malformations. In order to measure self-esteem, the Janis and Field Scale was used. The sample consisted of 608 adolescents aged 17 to 20 years including males and females. Two hundred and thirty-five had a cleft lip and/or palate and 373 did not. The analysis of the self-esteem levels obtained enabled to conclude that the adolescents with a cleft lip and/or palate behave differently from those without it, that is, they present lower self-esteem scores.

KEY WORDS: adolescence, self-esteem, cleft lip, cleft palate

\section{LA AUTOESTIMA DE ADOLESCENTES CON Y SIN FISURA DE LÁBIO YIO PALADAR}

La autoimagem es un determinante importante de la autoestima, entre hombres y mujeres, esto nos hace reflexionar sobre el proceso de rehabilitación de las personas portadoras de malformación. Utilizamos para medir el nivel de autoestima la escala de Janis e Field. Se entrevistaron 608 adolescentes de ambos sexos, con edades oscilantes entre 17 y 20 años y de ambos sexos, siendo 235 con fisura de labio y/o paladar y 373 sin esta situación. Analizando los niveles de autoestima concluimos que el grupo de adolescentes con fisuras se comporta de forma diferente al grupo sin fisuras, o sea, los portadores de fisuras exhiben menores escores de autoestima.

DESCRIPTORES: adolescencia, autoestima, fisura del labio, fisura del paladar

\footnotetext{
${ }^{1}$ Extraído da dissertação de mestrado: - A auto-estima em adolescentes com e sem fissuras de lábio e/ou de palato; ${ }^{2}$ Enfermeiro, Professor Doutor da Escola de Enfermagem de Ribeirão Preto da Universidade de São Paulo, Centro Colaborador da OMS para o desenvolvimento da pesquisa em enfermagem, e-mail: dandrade@eerp.usp.br; ${ }^{3}$ Professor Titular da Escola de Enfermagem de Ribeirão Preto da Universidade de São Paulo, Centro Colaborador da OMS para o desenvolvimento da pesquisa em enfermagem
} 
INTRODUÇÃO

A nossa experiência profissional com pessoas portadoras de malformações craniofaciais levou-nos a uma série de questionamentos e preocupações acerca do sentimento de autoestima. Nossas inquietações relacionam a presença de malformações com sentimentos de inferioridade que pode se agravar na adolescência, período da vida caracterizado por amplas e profundas modificações psicossomáticas.

Neste sentido, achamos oportuno resgatar...só contemplando os casos de mau formação congênita lábio-palatal, participando das reações emotivas e psicossomáticas de seus portadores, compreendemos a grandeza da estética dos seres humanos normais e a importância transcendental do complexo maxilofacial na concretização fisionômica ${ }^{(1)}$.

Incansáveis investigações permearam o fato de que os sujeitos portadores de fissuras orais congênitas enfrentam muitas barreiras para atingir um desenvolvimento psicológico satisfatório. Estas barreiras associadas ao desejo de ter um corpo perfeito, saudável e atraente repercutirão negativamente sobre a auto-imagem e, conseqüentemente sobre a auto-estima ${ }^{(2-5)}$, dentre outros.

Sob este prisma, complementamos ... a satisfação da necessidade de auto-estima leva o indivíduo a sentir-se confiante (no seu valor, força, capacidade e adequação) mais útil e necessário ao mundo. A não satisfação produz no indivíduo um sentimento de inferioridade, fraqueza e impotência. A persistência desses sentimentos desencadeará fracassos na sua trajetória ou processos patológicos variados ${ }^{(6)}$

$O$ conceito de auto-estima tem sido estudado e considerado como um importante indicador da saúde mental. A crítica, em geral, enfoca a necessidade de aplicação de instrumentos precisos que permita avaliar a necessidade de auto-estima de cada indivíduo.

Diante disso, é procedente a realização de nosso estudo que se caracterizou na avaliação dos níveis de auto-estima em adolescentes com e sem fissuras de lábio e/ou palato.

Acrescido às considerações acima, também, existe o fato de que nas últimas décadas a adolescência tem sido amplamente abordada na literatura por pesquisadores de diferentes áreas do conhecimento, no entanto, na enfermagem ainda existe escassez de artigos publicados ${ }^{(7)}$.

Por outro lado, é notória a escassez de pesquisas na enfermagem relacionadas a auto-estima e quando o tema proposto envolve adolescentes com malformação a situação torna-se ainda pior.

Neste contexto, o cuidado de enfermagem a pacientes com comprometimento da auto-estima, é destacado, principalmente, considerando que o enfermeiro é um agente qualificado para assistir o paciente no seu restabelecimento, pois, durante as ações do cuidar consegue, muitas vezes, descobrir a forma de pensar, os medos, as esperanças e as aspirações da clientela assistida ${ }^{(8)}$.

Por este motivo o enfermeiro precisa utilizar instrumentos que the permita seguramente avaliar e posteriormente, intervir de forma eficaz.

Todas as considerações feitas a respeito do cuidado de enfermagem, da adolescência, dos portadores de malformações e do sentimento de auto-estima se fizeram necessárias para complementar o ponto almejado deste estudo, que se seguem:

- verificar os índices de auto-estima dos adolescentes com e sem fissura de lábio e/ou de palato;

- comparar os níveis de auto-estima entre os sexos nos dois grupos de adolescentes estudados.

\section{MÉTODOS}

\section{População e Seleção da Amostra}

A população deste estudo constituiu-se de adolescentes com e sem fissuras de lábio e/ou de palato na faixa etária dos 17 aos 20 anos e de ambos os sexos. A amostra compôs-se de adolescentes portadores de fissuras de lábio e/ou de palato que já fizeram cirurgia estética e funcional e adolescentes sem fissuras.

Os jovens entrevistados sem fissuras foram localizados ao acaso em escolas públicas de $1^{\circ}$ e $2^{\circ}$ grau do interior do estado de São Paulo, e em suas residências. Já os portadores de malformação foram entrevistados no hospital que presta atendimento a este tipo de anomalia.

Procedimentos

Utilizou-se na coleta de dados a escala de auto-estima de Janis e Field que é uma tradução francesa adaptada no Brasil por Uchoa em 1976. É importante esclarecer que tal escala foi validada, portanto, considera-se adequada para medir aquilo que se propõe, $\mathrm{e}$ fidedigna, o que garante a confiabilidade dos resultados obtidos ${ }^{(9)}$.

A Escala de Janis e Field é do tipo Likert, exigindo uma resposta graduada para cada afirmação. $O$ instrumento é composto de 23 itens com alternativas graduadas de 1 a 5, ou seja, resposta equivalente a: completa aprovação (1), aprovação (2), neutralidade (3), desaprovação incompleta (4), desaprovação total (5). Apenas às 
questões 3 e 7 são creditados valores contrários aos mencionados, completa aprovação (5). A soma dos pontos representará a classificação do indivíduo que deverá ser interpretado em função de normas criteriosamente estabelecidas.

De acordo com a avaliação final ficou considerado o mais alto grau de pontuação 115, e o menor 23 pontos.

Procedimento ético na pesquisa com seres humanos

O estudo contou com o consentimento livre e esclarecido dos sujeitos da pesquisa, bem como, dos seus respectivos representantes legais. Utilizou-se todos os recursos que asseguram a confidencialidade, a privacidade, a proteção da imagem e a não estigmatização, garantindo a não veiculação das informações em prejuízo das pessoas envolvidas.

Plano Piloto

A aplicação desta escala foi submetida a um teste piloto envolvendo 30 adolescentes a fim de permitir a familiarização do pesquisador, e de verificar a compreensão das perguntas por parte dos sujeitos envolvidos no estudo. Por meio do teste piloto, também, planejamos a estratégia de coleta oficial dos dados.

Coleta oficial dos dados

O preenchimento da escala foi executado pelos sujeitos do estudo. Para o auto-preenchimento da escala respeitava-se o princípio da privacidade e da não interrupção. Aplicava-se o instrumento em grupos pequenos, até sete adolescentes por vez, ou individualmente. Para que o estudo tivesse credibilidade, enfatizou-se a importância da sinceridade no preenchimento da escala. Os adolescentes que participaram deste estudo mostraram-se, de uma forma geral, receptivos e interessados.

\section{RESULTADOS E DISCUSSÃO}

Em sua composição a amostra desse estudo está representada por um total de 235 adolescentes portadores de fissuras orais congênitas e 373 não portadores.

Estudo dos Níveis de auto-estima

Na Tabela 1 estão distribuídos os escores de auto-estima obtidos na amostra em estudo.
Tabela 1 - Distribuição dos adolescentes com e sem fissuras de lábio e/ou de palato de acordo com os níveis de auto-estima

\begin{tabular}{ccccccc}
\hline $\begin{array}{c}\text { Niveis de } \\
\text { Autoestima }\end{array}$ & \multicolumn{3}{c}{$\begin{array}{c}\text { Adolescentes } \\
\text { Com fissuras }\end{array}$} & \multicolumn{3}{c}{$\begin{array}{c}\text { Adolescentes } \\
\text { Sem fissuras }\end{array}$} \\
& $\mathrm{N}$ & $\%$ & $\mathrm{f}(\boldsymbol{\alpha}) \%$ & $\mathrm{~N}$ & $\%$ & $\mathrm{~F}(\boldsymbol{\alpha}) \%$ \\
\hline $27-37$ & 3 & 1,3 & 1,3 & 1 & 0,2 & 0,2 \\
$37-47$ & 27 & 11,5 & 12,8 & 7 & 1,9 & 2,1 \\
$47-57$ & 41 & 17,4 & 30,2 & 25 & 6,7 & 8,8 \\
$57-67$ & 68 & 28,9 & 59,1 & 65 & 17,4 & 26,2 \\
$67-77$ & 57 & 24,2 & 83,3 & 124 & 33,2 & 59,4 \\
$77-87$ & 28 & 11,9 & 95,2 & 89 & 23,9 & 83,3 \\
$87-97$ & 10 & 4,2 & 99,4 & 49 & 13,1 & 96,4 \\
$97-107$ & 1 & 0,4 & 100 & 13 & 3,5 & 100 \\
Total & 235 & 100 & & 373 & 100 & \\
\hline
\end{tabular}

Com base nos resultados obtidos em nosso estudo é possível concluir que os níveis de auto-estima obtidos são diferentes nos dois grupos estudados. A última coluna, isto é, da freqüência acumulada $\mathrm{f}(\mathrm{ac})$ nos permite concluir que $59 \%$ dos adolescentes com fissuras tem nível de auto estima abaixo de 67 , em relação $26,1 \%$ dos jovens sem fissuras.

Avaliação dos níveis de auto-estima de acordo com os sexos

Os estudos sobre o desenvolvimento do adolescente revelam que existem nítidas diferenças entre os sexos que ultrapassam as óbvias diferenças anatômicas ${ }^{(10-16)}$. No entanto, verificamos que há controvérsias nestes estudos quanto ao que produz tais diferenças, mas não há dúvidas de que elas existam (Tabela 2).

Tabela 2 - Distribuição dos níveis de auto-estima dos adolescentes com e sem fissuras de lábio e/ou de palato de acordo com os sexos

\begin{tabular}{|c|c|c|c|c|c|c|c|c|}
\hline \multirow{3}{*}{$\begin{array}{l}\text { Niveis de } \\
\text { Autoestima }\end{array}$} & \multicolumn{4}{|c|}{ Adolescentes com issuras } & \multicolumn{4}{|c|}{ Adolescentes sem issuras } \\
\hline & \multicolumn{2}{|c|}{ Mascuino } & \multicolumn{2}{|c|}{ Ferminino } & \multicolumn{2}{|c|}{ Mascuino } & \multicolumn{2}{|c|}{ Ferminino } \\
\hline & $N$ & $\%$ & $N$ & 8 & $N$ & 8 & $N$ & 8 \\
\hline $27 \cdot 37$ & 0 & 0 & 3 & 2,9 & 0 & 0 & 1 & 0,5 \\
\hline 37.47 & 11 & 8,2 & 16 & 15,6 & 2 & 1,1 & 5 & 2,7 \\
\hline $47 \cdot 57$ & 16 & 2,0 & 25 & 24,5 & 6 & 3,2 & 19 & 10,1 \\
\hline 57.67 & 46 & 34,5 & 22 & 21,5 & 31 & 16,6 & 34 & 18,1 \\
\hline 67.77 & 33 & 24,8 & 24 & 23,5 & 59 & 31,7 & 65 & 34,7 \\
\hline 77.87 & 18 & 13,5 & 10 & 98 & 52 & 27,9 & 37 & 1977 \\
\hline 87.97 & 8 & 6,0 & 2 & 1,9 & 26 & 13,9 & 23 & 12,2 \\
\hline $97 \cdot 107$ & 1 & 0,4 & 0 & 0 & 10 & 5,3 & 3 & 1,6 \\
\hline Total & 133 & 100 & 102 & 100 & 186 & 100 & 187 & 100 \\
\hline
\end{tabular}

Para verificarmos a influência do sexo (masculino fissurado $\left(M_{F}\right)$, feminino fissurado $\left(F_{F}\right)$; feminino fissurado $\left(F_{F}\right)$, feminino não fissurado $\left(F_{N}\right)$, masculino fissurado $\left(M_{F}\right)$ e masculino não fissurado $\left(\mathrm{M}_{\mathrm{N}}\right)$ sobre os níveis de auto-estima utilizamos o teste de MannWhitney ${ }^{(17)}$. De acordo com o referido teste verificamos que há influência do sexo no nível de auto-estima nos dois grupos considerados (com e sem fissuras), Tabela 3 e 4. 
Tabela 3 - Postos médios e resultados do teste estatístico não paramétrico de Mann-Whitney para o nível de auto-estima de adolescentes segundo o sexo no seu grupo de fissurado e não fissurado

\begin{tabular}{|c|c|c|c|c|}
\hline \multirow[t]{2}{*}{ Adolescentes } & \multicolumn{2}{|c|}{ Sexo } & \multirow{2}{*}{$\begin{array}{l}\text { Resultado do } \\
\text { teste }\end{array}$} & \multirow[t]{2}{*}{ Conclusã̃o } \\
\hline & Masculino & Feminino & & \\
\hline com fissura & 131,12 & 100,89 & $z=3,30 \quad p<0,01$ & $\neq \mathrm{F}_{\mathrm{r}}$ \\
\hline sem fissura & 204,92 & 169,17 & $z=3,20 \quad p \leq 0,01$ & $M_{\mathrm{N}} \neq \mathrm{F}_{\mathrm{N}}$ \\
\hline
\end{tabular}

Tabela 4 - Postos médios e resultados do teste estatístico não paramétrico de Mann-Whitney para o nível de auto-estima de adolescentes segundo o sexo no seu grupo de fissurado e não fissurado

\begin{tabular}{|c|c|c|c|c|}
\hline \multirow[t]{2}{*}{ Adolescentes } & \multicolumn{2}{|c|}{ Sexo } & \multirow{2}{*}{$\begin{array}{l}\text { Resultado do } \\
\text { teste }\end{array}$} & \multirow[t]{2}{*}{ Conclusão } \\
\hline & Masculino & Feminino & & \\
\hline com fissura & 102,41 & 168,23 & $z=6,40 \quad p \leq 0,01$ & $\mathrm{~F}_{\mathrm{r}}$ \\
\hline sem fissura & 120,22 & 188,45 & $z=6,51 \quad p \leq 0,01$ & $M_{r} \neq M_{N}$ \\
\hline
\end{tabular}

Conforme tabela acima, as adolescentes com fissuras $\left(\mathrm{F}_{\mathrm{F}}\right)$ diferem significativamente das não portadoras de fissuras. Tal afirmação, também, é válida para os jovens do sexo masculino. Os jovens portadores de fissura exibem os menores valores em comparação aos demais integrantes do estudo.

O estudo realizado em 1950 mostrou que as meninas, em geral, aspiravam ter corpos "bem contornados" e os meninos físicos mais "masculinos". Ambos os sexos confessaram suas preocupações com supostos "defeitos", manchas, acnes, nariz proeminente, dentes irregulares, sinais de nascimento e assim por diante ${ }^{(10)}$.

Outra investigação forneceu evidências adicionais para fundamentar esta alegada diferença sexual entre os adolescentes. Após entrevistar uma amostra considerável de adolescentes, os autores concluíram que as participantes femininas eram menos internalizadas e autônomas do que os sujeitos masculinos. Para ser mais precisa, as meninas eram mais passivas que os meninos $e$ relataram ter menos conflitos com os pais. Os meninos pareciam estar mais ativamente empenhados em formular seus próprios padrões de conduta do que simplesmente adotar indiscriminadamente valores de seus pais ${ }^{(18)}$.

Acredita-se que as meninas expressaram maior insatisfação do que os meninos com a aparência. Os autores justificavam este sentimento a partir da evidência de que as meninas experimentavam maior pressão social para ter uma aparência atraente. Parece que a

\section{REFERÊNCIAS BIBLIOGRÁFICAS}

1. Souza-Freitas JA. Centro de Pesquisa e Reabilitação de Lesões Lábio-Palatais: Manual de orientação. Bauru: Faculdade de Odontologia de Bauru da Universidade de São Paulo: 1974. aparência é especificamente mais importante para as mulheres do que para os homens ${ }^{(11)}$.

A partir daí, outros estudos, também corroboraram as diferenças psicossociais entre os sexos ${ }^{(16,19-20)}$ dentre outros.

\section{CONSIDERAÇÕES FINAIS}

Os estudos sobre auto-estima podem ser realizados a partir de diferentes pressupostos filosóficos e metodológicos. Optamos pelo uso de escala por ser uma forma de investigação crescente na enfermagem, além da possibilidade da aplicabilidade prática.

Os resultados obtidos quanto aos níveis de auto-estima demonstram diferenças significativas nos dois grupos considerados, isto é, o grupo de adolescentes portadores de fissuras de lábio e/ou de palato exibiram menores escores. Entretanto, as adolescentes com fissuras $\left(F_{F}\right)$ são significativamente diferentes das jovens sem fissuras.

Sem dúvida que estamos frente a um grupo de pessoas que apresenta distúrbios da auto-estima o que precisa ser considerado quando se almeja um cuidado de enfermagem efetivo.

$\mathrm{O}$ atendimento à pessoa portadora de malformação tem se mostrado em nosso país como uma área de trabalho que mantém uma grande demanda. No entanto, ao mesmo tempo, mostra-se como um segmento do mercado ao qual raramente os jovens profissionais encaminham sua opção de trabalho.

Quanto aos portadores de fissuras de lábio e/ou de palato, eles carregam consigo os seus defeitos e tudo o que socialmente este pode significar, fixando o defeito físico como parte de sua identidade. 0 que este estigma pode significar e ocasionar irá depender de vários fatores, uma questão subjetiva e reflexiva, podendo se constituir em um importante problema de ordem psicológica.

Dessa forma, é de suma importância que os integrantes da equipe de saúde desenvolvam seus trabalhos respaldados por um corpo de conhecimento cientificamente aceito para que possam dar uma assistência integral à clientela atendida e seus familiares.

\section{Agradecimento especial}

Ao Prof.Dr. Carlos Roberto Padovani do Instituto de Biociências do Campus da Universidade Estadual Paulista de Botucatu, pela valiosa assessoria estatística.

2. Richman LC. Self-reported social, speech, and facial concerns and personality adjustment of adolescents with cleft lip and palate. Cleft Palate J 1983 Apr; 20(2):108-12.

3. Leonard BJ, Brust JD, Abrahams G, Sielaff B. Self-concept of children and adolescents with cleft lip and/or palate. Cleft Palate Craniofac J 1991 Oct; 28(4):347-53. 
4. Kapp-Simon KA. Psychlogical interventions for the adolescents with cleft lip and palate. Cleft Palate Craniofac J 1995 Mar; 32(2):1048.

5. Sanches-Ruiz I, Gonzalez LG, Perez Gonzalez V, Diez Rodriguez R, Lopez-Cedrun JL, Miro Viar J, et al. Integrated treatment of cleft lip and palate: Organization of a treatment team. Cir Pediatr 1999 Jan; 12(1):4-10.

6. Maslow AH. Motivation and personality. $2^{\text {nd }}$ ed. New York (NY): Harper \& How; 1970.

7. Cano MAT, Ferriani MGC, Alves AC, Nakata CY. A produção do conhecimento sobre adolescência na enfermagem: período de 1983 a 1996. Rev Latino-am Enfermagem 1998 janeiro; 6(1):91-7.

8. Price B. A model for body image care. J Adv Nurs 1990;15:585-93.

9. Cardoso SES. Auto-estima e crime: base para o nexo entre delinqüência juvenil e aspectos da personalidade. [dissertação]. Rio de Janeiro (RJ): Pontífica Universidade Católica; 1979.

10. Frazier A, Lisobee LK. Adolescence concerns with physique. School Rev 1950; 58:387-405.

11. Dwyer J, Mayer J. Psychological effects of variations in physical appearance during adolescence. Adolescence 1969; 3:353-60.
12. Rosemberg M, Simmons RG. Disturbance in the self-image at adolescence. Am Sociol Rev 1973; 38:553-68.

13. Brantley HT, Clifford E. Cognitive self-concept and body image measure of normal. Cleft Palate J 1982; 16:177-82.

14. Bledson JC. Is self-concept a reliable predictor of economic status? Psychol Rep 1981; 49:883-6.

15. Richman LC, Eliason M. Psychological characteristics of children with cleft lip and palate: intelectual achievement, behavioral and personality variables. Cleft Palate J 1982; 19:249-57.

16. Bednar RL, Wells MG, Peterson SR. Self-esteem: paradoxes and innovation in clinical theory and practice. Washington, DC: American Psychological Association; 1989.

17. Siegel S, Castellan NJ. Nonparametrics statistics for the behavioral sciences. $2^{\text {nd }}$ ed. New York (NY): McGraw-Hill; 1988.

18. Jones MC, Mussen PH. Self-conceptions, motivations and interpersonal attitudes of early and later maturing girls. Child Dev 1958; 29:491-501.

19. Ribeiro MA. O autoconceito de adolescentes segundo o sexo e a estrutura familiar. Psicol Teor Pesqui 1988; 4:85-95.

20. Oliveira, MAC. Adolescência, 0 adolescer e 0 adolescente: resignificação a partir da determinação social do processo saúdedoença.[tese]. São Paulo(SP): Escola de Enfermagem/USP; 1997. 